PROCEEDINGS OF THE

AMERICAN MATHEMATICAL SOCIETY

Volume 136, Number 10, October 2008, Pages 3669-3673

S 0002-9939(08)09348-9

Article electronically published on May 19, 2008

\title{
FINITE UNIONS OF EQUIVALENCE RELATIONS
}

\author{
JOHN KITTRELL
}

(Communicated by Julia Knight)

\begin{abstract}
Say that a class of equivalence relations $\mathcal{C}$ has the finite union property if every equivalence relation that is the union of finitely many members of $\mathcal{C}$ must itself be a member of $\mathcal{C}$. Then the classes of hyperfinite, measureamenable, Fréchet-amenable, and cheap equivalence relations have the finite union property.
\end{abstract}

\section{INTRODUCTION}

Let $X$ be a standard Borel space. An equivalence relation $E$ on $X$ is hyperfinite if there is an increasing sequence $\left\langle F_{n}\right\rangle_{n \in \mathbb{N}}$ of finite Borel subequivalence relations of $E$ such that $E=\bigcup_{n \in \mathbb{N}} F_{n}$ (equivalently, if there is a Borel action of $\mathbb{Z}$ on $X$ that induces $E$ ). For more on countable Borel equivalence relations in general, see Jackson-Kechris-Louveau 2]. Despite being the object of much scrutiny in recent years, many basic questions about hyperfiniteness remain open. We answer one such question by showing that if there are hyperfinite equivalence relations $E_{1}, \ldots, E_{n}$ such that $E=\bigcup_{i \leq n} E_{i}$ is an equivalence relation, then $E$ is hyperfinite 1 Our proof will actually extend to wider classes of equivalence relations, so we state the theorem in a more general form.

Suppose $Y$ is a non-empty set and $E$ is an arbitrary equivalence relation on $Y$. A set $B \subseteq Y$ is $E$-invariant if

$$
(\forall x \in B)(\forall y \in Y) x E y \Rightarrow y \in B .
$$

A set $C \subseteq Y$ is a complete section of $E$ if $(\forall x \in Y)[x]_{E} \cap C \neq \emptyset$. If $F$ is also an equivalence relation on $Y$ and $E \subseteq F$, say that $E$ has finite index in $F$ if every $F$ class contains at most finitely many $E$-classes. If $\mathcal{C}$ is a class of equivalence relations, say that $\mathcal{C}$ has the finite union property if $E \in \mathcal{C}$ whenever there are equivalence relations $E_{1}, \ldots, E_{n} \in \mathcal{C}$ such that $\operatorname{dom}\left(E_{1}\right)=\cdots=\operatorname{dom}\left(E_{n}\right)$ and $E=\bigcup_{i \leq n} E_{i}$.

Theorem 1.1. Suppose that $\mathcal{C}$ is a class of countable Borel equivalence relations such that for $E$ and $F$ arbitrary countable Borel equivalence relations,

(1) if $\operatorname{dom}(E)=\operatorname{dom}(F), E \subseteq F, E \in \mathcal{C}$, and $E$ has finite index in $F$, then $F \in \mathcal{C}$

Received by the editors March 26, 2007, and, in revised form, September 12, 2007.

2000 Mathematics Subject Classification. Primary 03E15; Secondary 03E20.

Key words and phrases. Countable Borel equivalence relations, hyperfinite equivalence relations, union problems.

${ }^{1}$ This question was originally asked by Alexander Kechris and later brought to my attention by Benjamin Miller.

(C)2008 American Mathematical Society Reverts to public domain 28 years from publication 
(2) if $B \subseteq \operatorname{dom}(E)$ is Borel and $E \in \mathcal{C}$, then $E \mid B \in \mathcal{C}$;

(3) if $B \subseteq \operatorname{dom}(E)$ is a Borel complete section of $E$ and $E \mid B \in \mathcal{C}$, then $E \in \mathcal{C}$; and

(4) if $\left\{X_{i}\right\}_{i \leq N}$ is a partition of $\operatorname{dom}(E)$ into finitely many Borel E-invariant sets with $E \mid X_{i} \in \mathcal{C}$ for each $i \leq N$, then $E \in \mathcal{C}$.

Then $\mathcal{C}$ has the finite union property.

The key to proving Theorem 1.1 is a lemma about covering sets with equivalence classes. We therefore make a brief combinatorial excursion before embarking on the proof.

\section{THe LEMMA}

Fix a non-empty set $Y$. Observe that if $E, E_{1}, \ldots, E_{n}$ are equivalence relations on $Y$ such that $E=\bigcup_{i \leq n} E_{i}$, then for every $E$-class $C$ we have

$$
(\forall x \in C) \bigcup_{i \leq n}[x]_{E_{i}}=C .
$$

However, not all of the $E_{i}$ subequivalence classes in the above union may be necessary to cover $C$. Some classes may be contained in the union of others. It will be convenient to analyze this observation in a more general setting.

Definition 2.1. Suppose $A \subseteq Y$ is a non-empty set and $\mathscr{E}=\left\langle E_{1}, \ldots, E_{n}\right\rangle$, where each $E_{i}$ is an equivalence relation on $Y$. Say that a sequence $\alpha \in(\mathcal{P}(A))^{n}$ is an $\mathscr{E}$-minimal cover of $A$ if there are points $x_{1}, \ldots, x_{n} \in A$ such that

(1) $\alpha=\left\langle\left[x_{1}\right]_{E_{1}}, \ldots,\left[x_{n}\right]_{E_{n}}\right\rangle$,

(2) $A=\bigcup_{i \leq n}\left[x_{i}\right]_{E_{i}}$, and

(3) $(\forall i \leq n)\left[x_{i}\right]_{E_{i}} \nsubseteq \bigcup_{j \neq i}\left[x_{j}\right]_{E_{j}}$.

The goal is to show that there are at most finitely many $\mathscr{E}$-minimal covers of a set for a fixed $\mathscr{E}$. In the following remarks assume $A, E_{i}$, and $\mathscr{E}$ are as above.

First, we would like to "relativize" the notion of an $\mathscr{E}$-minimal cover to subsets of $A$. Informally speaking, the first remark below says that removing sets from an $\mathscr{E}$-minimal cover leaves an $\mathscr{E}^{\prime}$-minimal cover for the leftover portion of $A$, where $\mathscr{E}^{\prime}$ is a sequence of equivalence relations on $Y$ with $\ln \left(\mathscr{E}^{\prime}\right)<\operatorname{lh}(\mathscr{E})$. The second remark below says that $\mathscr{E}$-minimal covers of subsets of $A$ in the relative sense are uniquely determined by $\mathscr{E}$.

Notation. If $t=\left\langle i_{1}, \ldots, i_{m}\right\rangle$ is a subsequence of $\langle 1, \ldots, n\rangle$ and $\alpha=\left\langle a_{1}, \ldots, a_{n}\right\rangle$, let $\alpha \mid t$ denote the sequence $\left\langle a_{i_{1}}, \ldots, a_{i_{m}}\right\rangle$.

Remark 2.2. Let $n \geq 2$ and $x_{1}, \ldots, x_{n} \in A$ be such that $\left\langle\left[x_{1}\right]_{E_{1}}, \ldots,\left[x_{n}\right]_{E_{n}}\right\rangle$ is an $\mathscr{E}$-minimal cover of $A$. Fix $i \leq n$. Define the non-empty set $A_{i}=A \backslash\left[x_{i}\right]_{E_{i}}$. Then let

$$
\mathscr{E}_{i}=\left\langle E_{1}\left|A_{i}, \ldots, E_{n}\right| A_{i}\right\rangle \text { and } \alpha_{i}=\left\langle\left[x_{1}\right]_{E_{1}} \cap A_{i}, \ldots,\left[x_{n}\right]_{E_{n}} \cap A_{i}\right\rangle .
$$

If $t=\langle 1, \ldots, \hat{i}, \ldots, n\rangle$ (the sequence $\langle 1, \ldots, n\rangle$ with its $i$ th term deleted), then $\alpha_{i} \mid t$ is an $\mathscr{E}_{i} \mid t$-minimal cover of $A_{i}$. Indeed, $\alpha_{i} \mid t$ clearly satisfies conditions (1) and (2) of Definition 2.1 relative to $\mathscr{E}_{i} \mid t$ and $A_{i}$. For $j \leq n$, define the set

$$
r_{j}=\left[x_{j}\right]_{E_{j}} \backslash \bigcup_{l \neq j}\left[x_{l}\right]_{E_{l}} \text {. }
$$


Then $r_{j} \neq \emptyset$ by condition (3) of Definition 2.1 relative to $\mathscr{E}$ and $A$. Suppose now that $j$ is a term in $t$. Then $r_{j} \subseteq\left[x_{j}\right]_{E_{j}} \cap A_{i}$ and $r_{j} \nsubseteq\left[x_{l}\right]_{E_{l}}$ for any $l \neq j$. Hence

$$
\left[x_{j}\right]_{E_{j}} \cap A_{i} \not \subseteq \bigcup_{l \neq j, i}\left[x_{l}\right]_{E_{l}} \cap A_{i} .
$$

So $\alpha_{i} \mid t$ satisfies condition (3) of Definition 2.1 relative to $\mathscr{E}_{i} \mid t$ and $A_{i}$.

Remark 2.3. Suppose $B \subseteq A$ is a non-empty set and let

$$
\mathscr{E}_{B}=\left\langle E_{1}\left|B, \ldots, E_{n}\right| B\right\rangle .
$$

If there are points $x_{1}, \ldots, x_{n} \in A$ such that

$$
\alpha_{B}=\left\langle\left[x_{1}\right]_{E_{1}} \cap B, \ldots,\left[x_{n}\right]_{E_{n}} \cap B\right\rangle
$$

is an $\mathscr{E}_{B}$-minimal cover of $B$, then $\alpha_{B}$ is uniquely determined by the equivalence classes $\left[x_{1}\right]_{E_{1}}, \ldots,\left[x_{n}\right]_{E_{n}}$. That is to say, there is no other choice of equivalence classes $\left[x_{1}^{\prime}\right]_{E_{1}}, \ldots,\left[x_{n}^{\prime}\right]_{E_{n}}$ such that

$$
\alpha_{B}=\left\langle\left[x_{1}^{\prime}\right]_{E_{1}} \cap B, \ldots,\left[x_{n}^{\prime}\right]_{E_{n}} \cap B\right\rangle .
$$

Indeed, suppose there was an $x_{1}^{\prime} \in A$ (say) such that $\left[x_{1}^{\prime}\right]_{E_{1}} \neq\left[x_{1}\right]_{E_{1}}$ and

$$
\left[x_{1}^{\prime}\right]_{E_{1}} \cap B=\left[x_{1}\right]_{E_{1}} \cap B .
$$

Then this would contradict the fact that $\left[x_{1}^{\prime}\right]_{E_{1}} \cap\left[x_{1}\right]_{E_{1}}=\emptyset$ and $\left[x_{1}\right]_{E_{1}} \cap B \neq \emptyset$.

We now come to the key lemma.

Lemma 2.4. If $A \subseteq Y$ is a non-empty set and $\mathscr{E}=\left\langle E_{1}, \ldots, E_{n}\right\rangle$, where each $E_{i}$ is an equivalence relation on $Y$, then there are at most finitely many $\mathscr{E}$-minimal covers of $A$.

Proof. The proof is by induction on $n$. The base case $n=1$ is trivial (there is at most one way to cover a set with one $E_{1}$-class). Inductively, assume that the lemma holds at $n \geq 1$. That is, if $A^{\prime} \subseteq Y$ is a non-empty set and $\mathscr{E}^{\prime}$ is a sequence of equivalence relations on $Y$ of length $n$, then there are at most finitely many $\mathscr{E}^{\prime}$-minimal covers of $A^{\prime}$. Suppose $A \subseteq Y$ is non-empty and $\mathscr{E}=\left\langle E_{1}, \ldots, E_{n+1}\right\rangle$, where each $E_{i}$ is an equivalence relation on $Y$.

For a sequence $s$, let $\pi_{i}(s)$ denote the $i$ th term that occurs in $s$. Define the set

$$
\mathcal{S}=\{\alpha: \alpha \text { is an } \mathscr{E} \text {-minimal cover of } A\} .
$$

Fix $a \in A$. Then define the set

$$
\mathcal{S}_{i}=\left\{\alpha \in \mathcal{S}: a \in \pi_{i}(\alpha)\right\} .
$$

It follows that $\mathcal{S}=\bigcup_{i \leq n+1} \mathcal{S}_{i}$, as $a$ must belong to some term in $\alpha$, for any $\alpha \in \mathcal{S}$. Note that

$$
\left(\forall \alpha, \beta \in \mathcal{S}_{i}\right) \pi_{i}(\alpha)=\pi_{i}(\beta),
$$

since two components of the same partition sharing a point must be the same. So let $\left[x_{i}\right]_{E_{i}}$ be this common $i$ th coordinate of all $\alpha \in \mathcal{S}_{i}$.

We now use the induction hypothesis to show that $\mathcal{S}_{i}$ is finite. Fix $\alpha \in \mathcal{S}_{i}$. Say that there are points $x_{1}, \ldots, x_{n+1} \in A$ (including $x_{i}$ from above) such that

$$
\alpha=\left\langle\left[x_{1}\right]_{E_{1}}, \ldots,\left[x_{n+1}\right]_{E_{n+1}}\right\rangle .
$$


It suffices to show that there are only finitely many choices for the terms in $\alpha$ (we already know that there is only one choice for its $i$ th coordinate, namely $\left.\left[x_{i}\right]_{E_{i}}\right)$. Let $A_{i}=A \backslash\left[x_{i}\right]_{E_{i}} \neq \emptyset$. Then set

$$
\mathscr{E}_{i}=\left\langle E_{1}\left|A_{i}, \ldots, E_{n+1}\right| A_{i}\right\rangle \text { and } \alpha_{i}=\left\langle\left[x_{1}\right]_{E_{1}} \cap A_{i}, \ldots,\left[x_{n+1}\right]_{E_{n+1}} \cap A_{i}\right\rangle .
$$

If $t=\langle 1, \ldots, \hat{i}, \ldots, n+1\rangle$, then $\alpha_{i} \mid t$ is an $\mathscr{E}_{i} \mid t$-minimal cover of $A_{i}$ by Remark 2.2 . By the induction hypothesis, there are only finitely many choices for the terms in $\alpha_{i} \mid t$, as $\operatorname{lh}(t)=n$. By Remark 2.3, the terms in $\alpha_{i} \mid t$ are uniquely determined by the terms in $\alpha \mid t$, so there are only finitely many choices for the terms in $\alpha \mid t$. Since $\left[x_{i}\right]_{E_{i}}$ (the $i$ th coordinate of $\alpha$ ) is fixed, there are only finitely many choices for the terms in $\alpha$.

\section{THE PROOF}

Given equivalence relations $E_{1}, \ldots, E_{n}$ having a common domain, Lemma 2.4 allows us to specify finitely many $E_{i}$-classes contained in a particular type of set (viz., a set that can be minimally covered by the equivalence relations). If we return to the original scenario and think of this particular set as an $E$-class of an equivalence relation $E$ such that $E=\bigcup_{i \leq n} E_{i}$, an approach to proving Theorem 1.1 presents itself.

Proof of Theorem 1.1. Suppose $E$ is an equivalence relation such that $E=\bigcup_{i \leq n} E_{i}$, $E_{i} \in \mathcal{C}$ for each $i$, and $X=\operatorname{dom}\left(E_{1}\right)=\cdots=\operatorname{dom}\left(E_{n}\right)$. We must show that $\bar{E} \in \mathcal{C}$. Let $\mathscr{E}=\left\langle E_{1}, \ldots, E_{n}\right\rangle$. The idea is to break $X$ into finitely many Borel $E$-invariant pieces corresponding to the "lexicographically least" minimal cover of a point's $E$-class, then show that the set of points that induce these covers is a complete section of $E$ on which the restriction of $E$ belongs to $\mathcal{C}$. Let $x \in X$ and $t \in \mathrm{Seq}_{n}$ be arbitrary for the remainder of the paragraph, where

$$
\mathrm{Seq}_{n}=\{t: t \neq \emptyset \text { is a subsequence of }\langle 1, \ldots, n\rangle\} .
$$

Define the sequence

$$
\alpha(x)=\left\langle[x]_{E_{1}}, \ldots,[x]_{E_{n}}\right\rangle .
$$

Let $t_{x}$ denote the lexicographically least $t$ such that

$$
\left(\exists y \in[x]_{E}\right) \alpha(y) \mid t \text { is an } \mathscr{E} \mid t \text {-minimal cover of }[x]_{E} .
$$

Note that $t_{x}$ is $E$-invariant. Define the Borel set

$$
X_{t}=\left\{y \in X: t_{y}=t\right\} .
$$

One approach to seeing that sets of this form are Borel is by using the FeldmanMoore Theorem [1] to define them in a first order way using only number quantifiers and Borel relations. Moreover, $X_{t}$ is $E$-invariant. We denote the collection of lexicographically least minimal covers of $[x]_{E}$ as

$$
Q_{x}=\left\{\alpha:\left(\exists y \in[x]_{E}\right) \alpha=\alpha(y) \mid t_{x} \text { is a } \mathscr{E} \mid t_{x} \text {-minimal cover of }[x]_{E}\right\} .
$$

Then $Q_{x}$ is finite by Lemma 2.4 and is $E$-invariant. Finally, define the Borel set

$$
C_{t}=\left\{y \in X_{t}: \alpha(y) \mid t \in Q_{y}\right\} .
$$

Informally, a point belongs to $C_{t}$ if it induces the correct minimal cover of the $E$-class to which it belongs. 
Let $t \in \mathrm{Seq}_{n}$ be such that $X_{t} \neq \emptyset$. Observe that $C_{t}$ is a Borel complete section of $E \mid X_{t}$. Indeed, fix $x \in X_{t}$. If $y \in[x]_{E}$, then

$$
[x]_{E}=\bigcup_{i \leq n}[y]_{E_{i}}
$$

and so there will be a collection of sequences $s \in \operatorname{Seq}_{n}$ such that $\alpha(y) \mid s$ is an $\mathscr{E} \mid s$ minimal cover of $[x]_{E}$ (just throw away the terms in $\alpha(y)$ that are not needed to cover $\left.[x]_{E}\right)$. Take the $\leq_{\text {lex }}$-infimum of all such sequences $s$ as $y$ varies over $[x]_{E}$. This infimum will be $t$, and the non-empty set of points $y \in[x]_{E}$ such that $\alpha(y) \mid t$ is an $\mathscr{E} \mid t$-minimal cover of $[x]_{E}$ will be $[x]_{E} \cap C_{t}$.

We now show that $E \mid C_{t} \in \mathcal{C}$. Let $i$ be a term in $t$, say the $j$ th one. By properties (1) and (2), it suffices to show that $E_{i} \mid C_{t}$ has finite index in $E \mid C_{t}$. Fix $x \in C_{t}$ and say $Q_{x}=\left\{\beta_{1}, \ldots, \beta_{k}\right\}$. Note that

$$
\left(\forall y \in[x]_{E}\right) \alpha(y) \mid t=\beta_{l} \Rightarrow y \in \pi_{j}\left(\beta_{l}\right) .
$$

So the set $\left\{y \in[x]_{E}: \alpha(y) \mid t=\beta_{l}\right\}$ is contained in one $E_{i}$-class; namely, $\pi_{j}\left(\beta_{l}\right)$. Seeing it as

$$
[x]_{E \mid C_{t}}=\bigcup_{l \leq k}\left\{y \in[x]_{E}: \alpha(y) \mid t=\beta_{l}\right\},
$$

the above observation shows that $[x]_{E \mid C_{t}}$ contains at most $k$-many $E_{i} \mid C_{t}$-classes.

It follows that $E \mid C_{t} \in \mathcal{C}$. Hence $E \mid X_{t} \in \mathcal{C}$ by property (3), as $C_{t}$ is a complete section of $E \mid X_{t}$. Since the non-empty $X_{t}$ sets partition $X$, we have $E \in \mathcal{C}$ by property (4).

Corollary 3.1. The classes of hyperfinite, measure-amenable, Fréchet-amenable and cheap equivalence relations have the finite union property.

Proof. It suffices to show that each class satisfies properties (1)-(4) in Theorem 1.1. The hyperfinite case follows from some applications of the Feldman-Moore Theorem 1]. See Proposition 1.3 of Jackson-Kechris-Louveau [2], for example. Proofs of the relevant closure properties for the amenable classes can also be found in JacksonKechris-Louveau (Propositions 2.9 and 2.15). See Section 26 of Kechris-Miller 3] for the case of cheap equivalence relations.

Remark 3.2. It is an open question whether the class of treeable equivalence relations (namely, those countable Borel equivalence relations that admit a tree structure on their equivalence classes) satisfies property (2) in Theorem 1.1. An affirmative answer would imply that this class also has the finite union property.

\section{REFERENCES}

[1] J. Feldman and C.C. Moore, Ergodic equivalence relations and von Neumann algebras, I, Trans. Amer. Math. Soc. 234 (1977), 289-324. MR0578656 (58:28261a)

[2] S. Jackson, A.S. Kechris, A. Louveau, Countable Borel equivalence relations, J. Math. Logic 2(1) (2002), 1-80. MR1900547 (2003f:03066)

[3] A.S. Kechris, B.D. Miller, Topics in orbit equivalence, Springer, 2004. MR 2095154 (2005f:37010)

Knightsbridge Asset Management, LlC, Suite 460, 660 Newport Center Drive, Newport Beach, California 92660

E-mail address: jw.kittrell@gmail.com 